EPJ Web of Conferences 97,00021 (2015)

DOI: 10.1051/epjconf/20159700021

(C) Owned by the authors, published by EDP Sciences, 2015

\title{
Production of Resonances Using CLAS at Jefferson Lab
}

\author{
Kei Moriya ${ }^{1, a}$ \\ for the CLAS Collaboration \\ ${ }^{1}$ Arizona State University
}

\begin{abstract}
Measurements of hadronic resonances produced in photoproduction reactions at Jefferson Lab are shown and discussed. Emphasis is placed on the production of the excited hyperon states $\Sigma(1385), \Lambda(1405)$, and $\Lambda(1520)$. Some future prospects for the upcoming Jefferson Lab $12 \mathrm{GeV}$ era are given, where the CLAS12 and GlueX detectors will see unprecedented amounts of data using electromagnetic probes and further our knowledge of hadronic resonances.
\end{abstract}

\section{Introduction}

The Thomas Jefferson National Accelerator Facility, commonly known as Jefferson Lab, has a broad research program that spans multiple areas of nuclear and particle physics. The CEBAF accelerator has up to now provided electron beam bunches with energies up to $6 \mathrm{GeV}$ every $2 \mathrm{~ns}$, and is the foundation that has allowed many experiments to produce world-class results using electromagnetic probes. Among these results are measurements of hadronic resonances, which have been produced with both electron and real photon beams. In this article we give a brief overview of the photoproduction program at Jefferson Lab and a review of what has been learned.

\section{CLAS Measurements of Hadronic Resonances}

The CLAS detector has been housed in Hall B at Jefferson Lab, and allows for the detection and reconstruction of multi-particle final states. In hadronic spectroscopy the goal is to map out and understand the spectrum of hadronic resonances, and this has been one of the main pillars of the CLAS physics program. The energies accessible at Jefferson Lab are ideally suited for studying the ground states of various hadrons as well as the rich spectra of excited states. The simplest classification of hadrons is into spin-integer mesons and spin-half-integer-baryons; however, their associated production mechanisms do not allow such simple divisions and often the physics are intertwined. Below we categorize the results from the CLAS detector based on the final state produced, but the physics of interest at times may be more in the reaction mechanism and the intermediate states that the reaction goes through.

\footnotetext{
ae-mail: kmoriya@jlab.org
} unrestricted use, distribution, and reproduction in any medium, provided the original work is properly cited. 


\subsection{Non-strange Mesons}

CLAS has produced photoproduction results of mesons such as single $\pi, \eta, \eta^{\prime}, \omega$, and $\phi$ production [1]. Various physics observables are accessible in these reactions, and the interests are bountiful [2,3]. For pseudoscalar production, coupling to intermediate $N^{*}$ states through the reaction

$$
\gamma+p \rightarrow\left(N^{*}\right) \rightarrow P+N,
$$

where $P$ and $N$ are respectively the pseudoscalar meson and the final state nucleon that decay from the intermediate $N^{*}$ state has attracted much attention. Measurements in polarized beam and/or target experiments give access to various asymmetries, and these can be used to further pin down expected behavior of resonance contributions.

For vector meson production, the spin density matrix elements of the resulting vector mesons are measurable through the decay distributions, revealing information on the production mechanisms and possible intermediate couplings. For polarized experiments again asymmetries are accessible and allow for further comparisons to theoretical models.

\subsection{Ground State Hyperons}

CLAS has published many results on ground state hyperon photoproduction [4]. Again one of the interests is to find intermediate $N^{*}$ states that couple through

$$
\gamma+p \rightarrow\left(N^{*}\right) \rightarrow K^{+}+Y
$$

where $Y$ represents a hyperon with strangeness $S=-1$ that couples to intermediate $N^{*}$ states. Many $N^{*}$ states have been claimed in the past and the PDG [5] keeps a comprehensive list of these states. Traditionally the $N^{*}$ states have been identified through experimental results from $\pi N$ scattering experiments, but high statistics data from modern electro- and photoproduction experiments now are of better quality and quantity to search for such states [2]. The current PDG has changed quite significantly from previous issues, and much of this change has been induced by this new data and the partial wave analyses that are applied to them [3]. Ref. [6] gives a comprehensive review of our current understanding of baryons and future directions.

Another interesting avenue being pursued at CLAS and other facilities is the possibility of complete measurements. With enough observables, the reactions of type Eq. (2) can be completely determined to the amplitude level so that all amplitude strengths and relative phases are determined. Several experiments with various polarization combinations for beam and target have already been taken, and together with polarization measurements of the decaying ground state hyperon, a complete measurement is possible. Several of these preliminary results have already been shown [7], and will be an exciting development as results are finalized. The various asymmetries measured in the final states of $\pi N, \eta N, \omega N, K^{+} \Lambda$ and $K^{+} \Sigma^{0}$ will provide further constraints to model the reaction mechanisms.

\section{$2.3 \Sigma(1385)$}

It was noted in a previous talk on STAR results at this workshop [8] that the $\Sigma(1385)$ invariant mass spectrum, or line shape, was found to be symmetric and was not modeled well with a mass-dependent width, in contrast to the $\Delta$ resonance that is. The mass-dependent width of a Breit-Wigner function is usually modeled in the form of:

$$
\Gamma(m)=\Gamma_{0}\left(\frac{q(m)}{q_{0}}\right)^{2 L+1},
$$


where $\Gamma(m)$ is the mass-dependent width for mass $m$ of the resonance. For a two-body decay of a resonance, $q$ is the magnitude of momentum of each daughter particle in the rest frame of the resonance, and $L$ is the breakup angular momentum determined from conservation of angular momentum and parity. The values of $q_{0}$ and $\Gamma_{0}$ are the breakup momentum and width, respectively, at the central mass of the resonance. The main modifications due to these factors is to skew the resonance asymmetrically to the high mass side since the breakup momentum increases with resonance mass, compared to a mass-independent width that has a symmetric shape. This is shown in Fig. 1.

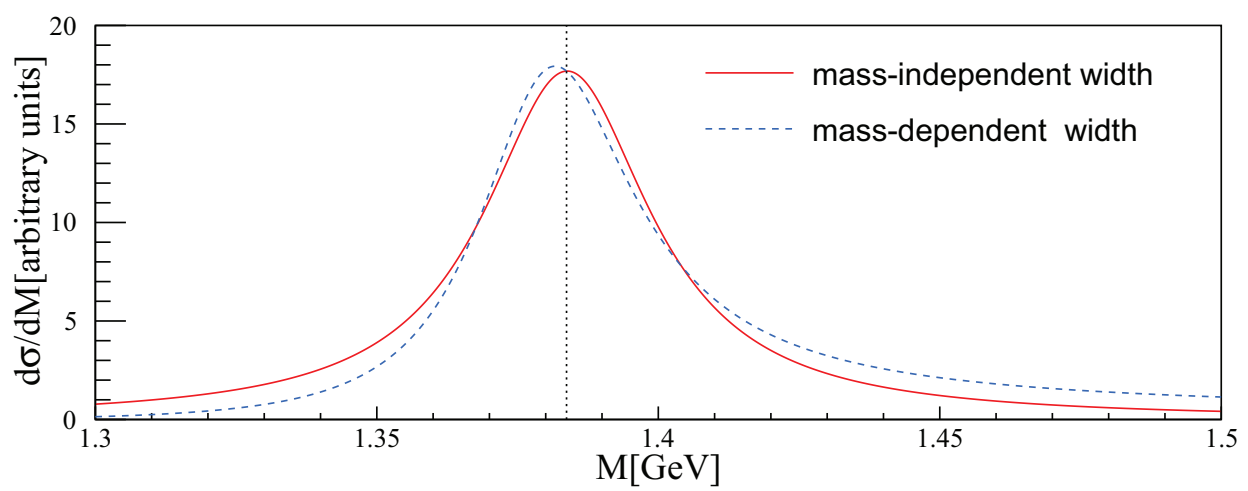

Figure 1: Relativistic Breit-Wigner functions with mass-independent width (red solid line) and massdependent width (dashed blue line). The vertical dotted line show the nominal peak position of the resonance. The mass-dependent function is skewed toward the higher mass.

It is notable that for the $\Sigma(1385)$ the mass-dependent form of the Breit-Wigner function has been found to be an inadequate description of experimental data in various reactions. The best measurements of the $\Sigma(1385)$ mass according to the PDG [5] are several bubble chamber experiments from negative kaon beam reactions. In all of these experiments a fit with Breit-Wigner functions containing mass-dependent widths were attempted but modifications were necessary to make the fit function symmetric [9]. Besides the STAR results from heavy ion collisions, HADES has also discussed this point for $\Sigma(1385)$ produced in $p-p$ collisions [10]. Another example is from CLAS which showed in Ref. [11] that in photoproduction the $\Sigma(1385)$ has a symmetric line shape that is better modeled with a mass-independent width. Figure 2 shows the $\Sigma(1385)$ line shape measured in the decay channel $\Lambda+\pi^{0}$ by CLAS. It remains a puzzle why this state fails to conform to expectations; some have suggested that there is a $1 / 2^{-} \Sigma^{*}$ state present in the same mass range to alter the line shape [12].

\section{$2.4 \Lambda(1405)$}

The $\Lambda(1405)$ state has been a controversial state for many decades, due to its peculiar line shape. This has been observed in past experiments [13,14] as well as more recent ones [15-17], and while it is generally agreed that this is due to its strong coupling to the $N \bar{K}$ final state that is above its nominal mass, the reason for this strong coupling has been a subject of debate. Chiral unitary theory, which combines chiral Lagrangians with unitarity among coupled channels, has been active in investigations of the $\Lambda(1405)$, and many theorists in this community think that the $\Lambda(1405)$ is a "dynamically generated" resonance that is generated through meson-baryon rescattering interactions. Also, it has been pointed out that there may be two poles in the vicinity of the $\Lambda(1405)$, and that different reaction 

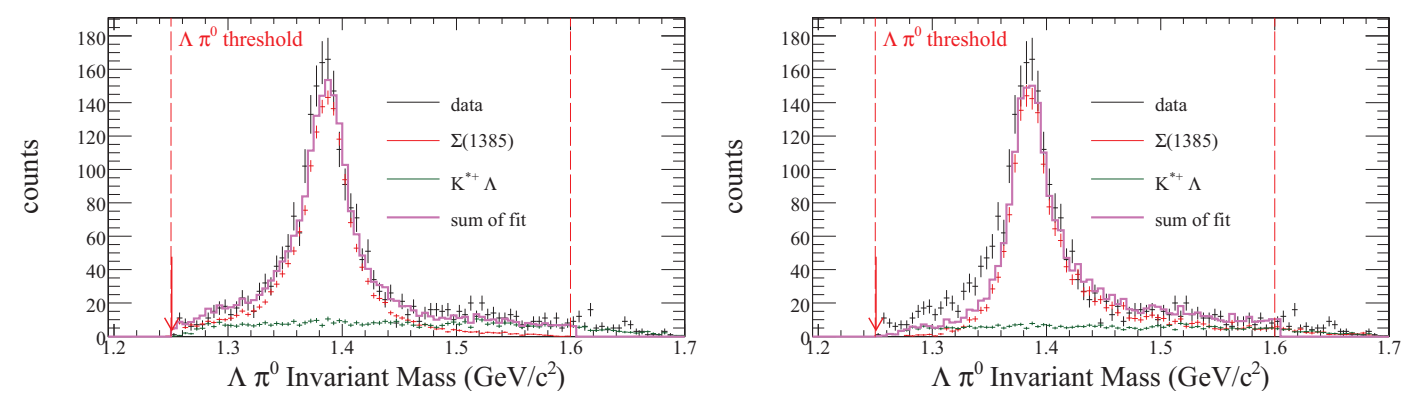

Figure 2: Invariant mass spectra of the $\Sigma^{0}(1385)$ peak using Monte Carlo templates based on (a) massindependent (b) mass-dependent relativistic Breit-Wigner forms. The fit with the mass-dependent Breit-Wigner form clearly does not fit the data well. Figures from Ref. [11].

mechanisms may excite the two poles in different ways which will lead to a production mechanismdependent line shape. in For a review of chiral unitary theory and the $\Lambda(1405)$, see Ref. [19].

The line shapes and differential cross section of the $\Lambda(1405)$ in photoproduction has been measured at CLAS $[11,20]$. Figure 3 shows an example of the measured line shape for a given center-ofmass energy range. The three colors depict the different $(\Sigma \pi)^{0}$ channels that the $\Lambda(1405)$ decays to. Clearly the line shapes are qualitatively different, and show different peak positions.

In response to the CLAS measurements, theorists in the chiral unitary community have published results that aim to model the results. Oset and Roca [22] show that a model based on the chiral unitary amplitudes is able to fit and reproduce the data well, and extract the two poles associated with the $\Lambda(1405)$. Nakamura and Jido [23] take into account many different reaction mechanisms to model the production of the $\Lambda(1405)$ followed by rescattering of meson-baryon interactions based on chiral unitary dynamics. The model is able to reproduce the line shapes of the three $\Sigma \pi$ channels, but further work is needed to model the differential cross sections. Investigations on whether different poles are excited for different reactions have been done for $p$ - $p$ reactions in Ref. [18] and also in Ref. [21] for electroproduction.

\subsection{Cross Sections of Hyperon Resonances}

Finally, a measurement of the photoproduction differential cross sections of the $\Sigma(1385), \Lambda(1405)$, and $\Lambda(1520)$ has been performed using the same CLAS dataset [20]. Although the natures of the three excited hyperons are quite different, as are their spin-parity quantum numbers, the overall behavior is similar, with all three states showing a forward peak that becomes more prominent with increasing energy (Fig. 4). The forward peak suggests a $t$-channel exchange mechanism dominating the production mechanism, and the magnitude of the cross sections are also similar.

The results of these measurements have spurred discussions again on intermediate nucleon resonances that could couple to the final state of $K Y^{*}$ with $Y^{*}$ being the $\Sigma(1385)$ or $\Lambda(1520)$ [24-26]. In particular, two studies $[25,26]$ show that the existence of a $N^{*}(2120)$ state is preferred to obtain an adequate description of the data. 

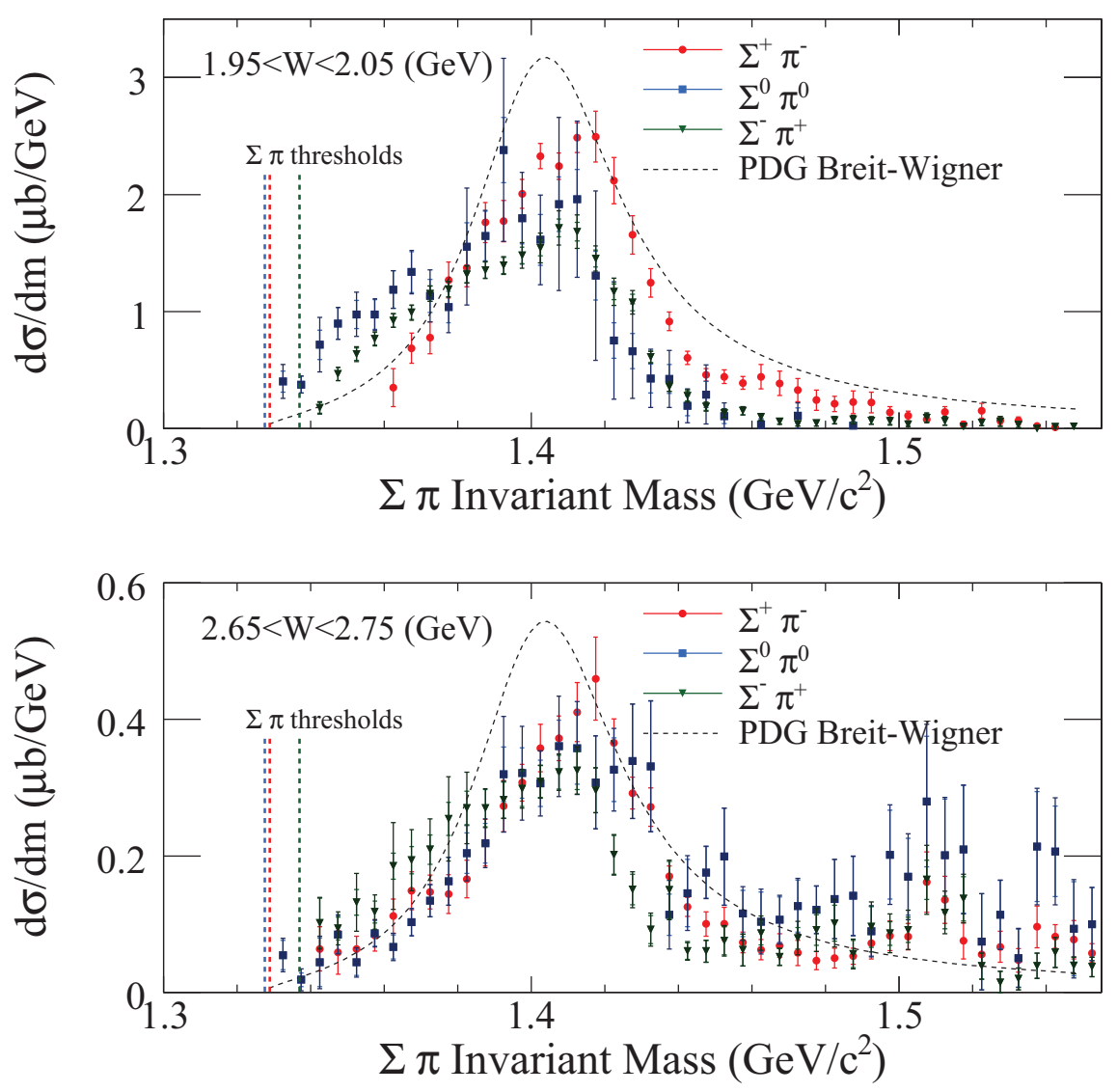

Figure 3: Invariant mass spectra of the $\Lambda(1405)$ for the three different decay modes of $(\Sigma \pi)^{0}$. The legends show each mode. (a) Low center-of-mass energy near production threshold. (b) High centerof-mass energy of $2.8 \mathrm{GeV}$. Figures from Ref. [11].

\section{The Jefferson Lab $12 \mathrm{GeV}$ Era}

Jefferson Lab is currently upgrading its accelerator energy to a maximum of $12 \mathrm{GeV}$, and each of the existing experimental halls are upgrading their detectors. The CLAS detector has been decommissioned, and a new CLAS12 detector is under construction. It will have multi-particle acceptance for the reconstruction of final state particles, and will be able to detect particles down to very forward angles. Proposals have been put forward and approved for the study of hadronic resonances. Ref. [27] proposes to study the excited meson spectrum using CLAS12, while Ref. [28] proposes the study of the strangeness $S=-2$ and -3 states. An overview of the nucleon structure program for CLAS12 can be found in Refs. [29].

A new experimental Hall D has been constructed, and houses the GlueX detector, which is shown schematically in Fig. 5. The main goal of the GlueX experiment will be to map out all accessible mesons in photoproduction and to search for mesons with quantum numbers that are not permitted using only quark-antiquark degrees of freedom. Excited strange baryon states with strangeness -1 and -2 will also be one of the main physics interests. Details of the expected physics, yields, as well as a 

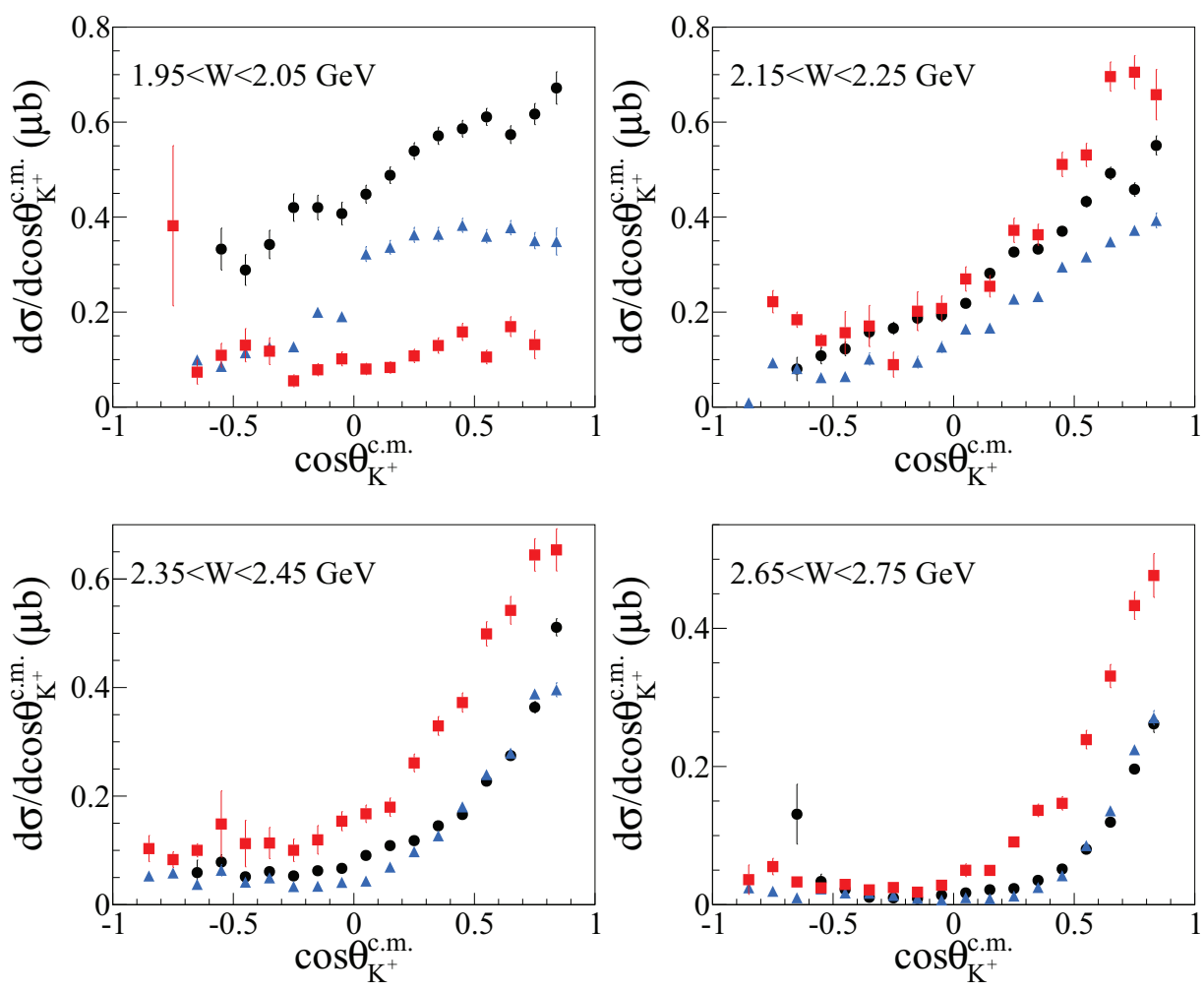

Figure 4: Differential cross sections of the $\Sigma(1385)$ (black circles), $\Lambda(1405)$ (blue triangles), and $\Lambda$ (1520) (red squares) for four center-of-mass energy ranges. Figures from Ref. [20].

discussion of the detectors can be found in Ref. [30]. Coincidentally, commissioning of the beam and detector systems were just beginning at the time of this workshop. After more commissioning in the spring of 2015, production datataking will begin, and this will lead to an unprecedented high-statistics dataset for photoproduction.

The primary goal of the GlueX experiment will be to search for so-called exotic mesons, mesons that have quantum numbers that cannot be explained by a simple $q \bar{q}$ system and necessitates other degrees of freedom. Other studies will be undertaken for hyperon resonances. For further details of both the CLAS12 and GlueX projects, we refer to Ref. [31].

\section{Summary}

The CLAS detector at Jefferson Lab has produced many photoproduction results related to hadronic resonances in the past decade. The high-statistics measurements have contributed to our knowledge of various aspects of hadronic physics. One area where the CLAS data has had a large impact is in advancing our understanding of the $N^{*}$ states. More results from various beam/target polarization asymmetry measurements will be appearing in the near future, with hopes of an amplitude-level understanding of the production mechanism of various final states. Finally, the production of excited hyperons such as the $\Lambda(1405), \Sigma(1385)$, and $\Lambda(1520)$ allows to study the characteristics of these states. 


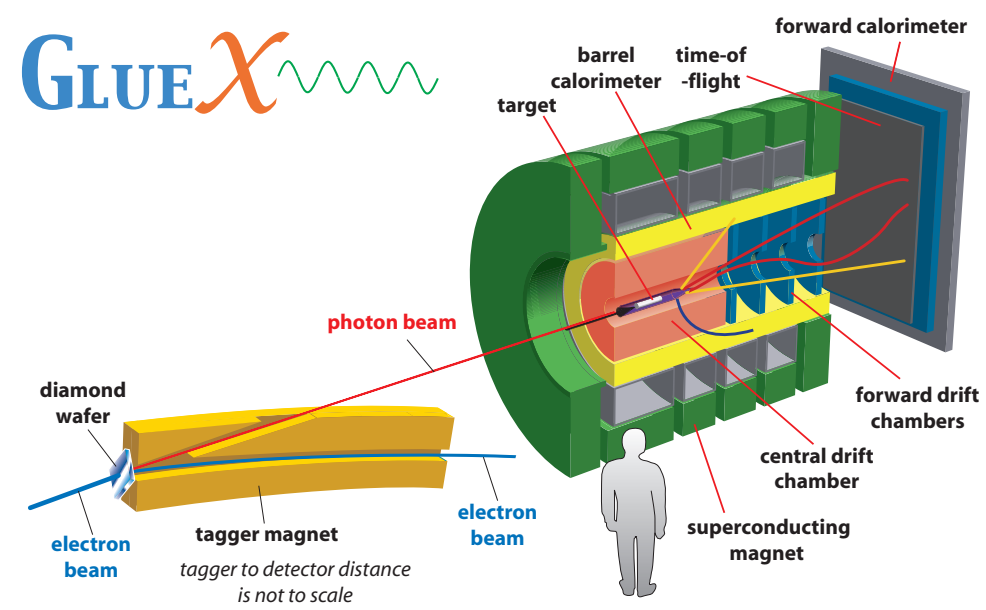

Figure 5. Schematic of the GlueX detector and photon tagging system.

CLAS has also published results on the strangeness $-2 \Xi$ states in photoproduction [32], but much more is expected in the future in the $12 \mathrm{GeV}$ era. The CLAS12 and GlueX detectors will operate under higher luminosities, a higher energy range, and better acceptances than the CLAS detector, and provide even more information on various hadronic resonances. The overall goal will be to contribute to our understanding of the spectrum of hadrons, and to find a path to connect the experimental measurements to the fundamental theory of QCD.

\section{Acknowledgments}

I would like to thank the Jefferson Science Association Junior Scientist Travel Support for their financial support, and to the organizers of this workshop for their hospitality.

\section{References}

[1] M. Dugger et al. [CLAS Collaboration], Phys. Rev. C 88, no. 6, 065203 (2013) [Addendum-ibid. C 89, no. 2, 029901 (2014)], M. Williams et al., Phys. Rev. C 80, 045213 (2009). M. Williams et al., Phys. Rev. C 80, 065208 (2009), B. Dey et al., Phys. Rev. C 89, no. 5, 055208 (2014) [Addendum-ibid. C 90, no. 1, 019901 (2014)].

[2] V. D. Burkert, H. Kamano, E. Klempt, D. Rönchen, A. V. Sarantsev, T. Sato, A. Svarc and L. Tiator et al., arXiv:1412.0241 [nucl-ex], I. G. Aznauryan and V. D. Burkert, Prog. Part. Nucl. Phys. 67, 1 (2012), I. Aznauryan, V. D. Burkert, T.-S. H. Lee and V. I. Mokeev, J. Phys. Conf. Ser. 299, 012008 (2011).

[3] A. V. Anisovich, R. Beck, E. Klempt, V. A. Nikonov, A. V. Sarantsev and U. Thoma, Eur. Phys. J. A 48, 15 (2012), A. V. Anisovich, R. Beck, V. Burkert, E. Klempt, M. E. McCracken, V. A. Nikonov, A. V. Sarantsev and R. A. Schumacher et al., Eur. Phys. J. A 50, 129 (2014)

[4] J. W. C. McNabb et al. [CLAS Collaboration], Phys. Rev. C 69, 042201 (2004), R. Bradford et al., Phys. Rev. C 73, 035202 (2006), R. K. Bradford et al., Phys. Rev. C 75, 035205 (2007), M. E. McCracken et al., Phys. Rev. C 81, 025201 (2010), B. Dey et al., Phys. Rev. C 82, 025202 (2010).

[5] K. A. Olive et al. [Particle Data Group Collaboration], Chin. Phys. C 38, 090001 (2014).

[6] V. Crede and W. Roberts, Rept. Prog. Phys. 76, 076301 (2013). 
[7] See for example the following from the CLAS Collaboration: R. Tucker et al., Int. J. Mod. Phys. Conf. Ser. 26, 1460078 (2014), E. Pasyuk, Int. J. Mod. Phys. Conf. Ser. 26, 1460083 (2014), D. Martinez et al., Int. J. Mod. Phys. Conf. Ser. 26, 1460103 (2014), T. Kageya, Int. J. Mod. Phys. Conf. Ser. 26, 1460079 (2014), N. K. Walford et al., Int. J. Mod. Phys. Conf. Ser. 26, 1460098 (2014), I. Senderovich et al., Int. J. Mod. Phys. Conf. Ser. 26, 1460115 (2014), B. G. Ritchie, EPJ Web Conf. 73, 04010 (2014), T. Kageya, Phys. Part. Nucl. 45, 135 (2014).

[8] Richard Witt, talk given at this workshop. For details see B. I. Abelev et al. [STAR Collaboration], Phys. Rev. Lett. 97, 132301 (2006), Phys. Rev. C 78, 044906 (2008).

[9] W. Cameron et al. [Rutherford-London Collaboration], Nucl. Phys. B 143, 189 (1978), S. R. Borenstein, G. R. Kalbfleisch, R. C. Strand, V. VanderBurg and J. W. Chapman, Phys. Rev. D 9, 3006 (1974), S. O. Holmgren et al. [Amsterdam-CERN-Nijmegen-Oxford Collaboration], Nucl. Phys. B 119, 261 (1977).

[10] G. Agakishiev et al. [HADES Collaboration], Phys. Rev. C 85, 035203 (2012).

[11] K. Moriya et al. [CLAS Collaboration], Phys. Rev. C 87, no. 3, 035206 (2013).

[12] Y. H. Chen and B. S. Zou, Phys. Rev. C 88, no. 2, 024304 (2013), P. Gao, J. J. Wu and B. S. Zou, Phys. Rev. C 81, 055203 (2010).

[13] D. W. Thomas, A. Engler, H. E. Fisk and R. W. Kraemer, Nucl. Phys. B 56, 15 (1973),

[14] R. J. Hemingway, Nucl. Phys. B 253, 742 (1985).

[15] I. Zychor et al., Phys. Lett. B 660, 167 (2008)

[16] M. Niiyama et al., Phys. Rev. C 78, 035202 (2008).

[17] G. Agakishiev et al. [HADES Collaboration], Phys. Rev. C 87, no. 2, 025201 (2013).

[18] J. Siebenson and L. Fabbietti, Phys. Rev. C 88, no. 5, 055201 (2013).

[19] T. Hyodo and D. Jido, Prog. Part. Nucl. Phys. 67, 55 (2012).

[20] K. Moriya et al. [CLAS Collaboration], Phys. Rev. C 88, 045201 (2013) [Addendum-ibid. C 88, no. 4, 049902 (2013)].

[21] H. Y. Lu et al. [CLAS Collaboration], Phys. Rev. C 88, 045202 (2013) [arXiv:1307.4411].

[22] Eulogio Oset, talk given at this workshop. L. Roca and E. Oset, Phys. Rev. C 88, no. 5, 055206 (2013), L. Roca and E. Oset, Phys. Rev. C 87, no. 5, 055201 (2013).

[23] S. X. Nakamura and D. Jido, PTEP 2014, 023 D01 (2014).

[24] J. He, Phys. Rev. C 89, 055204 (2014).

[25] J. He, Nucl. Phys. A 927, 24 (2014).

[26] J. J. Xie, E. Wang and J. Nieves, Phys. Rev. C 89, no. 1, 015203 (2014).

[27] Marco Battaglieri et al. [CLAS12 Collaboration], Proposal to Jefferson Lab Program Advisory Committee, https://www.jlab.org/exp_prog/proposals/11/PR12-11-005.pdf (2011).

[28] A. Afanassev et al. [CLAS12 Collaboration], Proposal to Jefferson Lab Program Advisory Committee, available at https://www.jlab.org/exp_prog/proposals/13/PR12-12-008. VeryStrangeUpdate.pdf (2012).

[29] V. D. Burkert, Nuovo Cim. C 036, no. 05, 259 (2013), V. D. Burkert, Nuovo Cim. C 035N2, 295 (2012), I. G. Aznauryan et al., Int. J. Mod. Phys. E 22, 1330015 (2013).

[30] GlueX proposals to Jefferson Lab Program Advisory Committee: http://arxiv.org/abs/1408.0215 (2014), http://arxiv.org/abs/1305.1523 (2013), http://arxiv.org/abs/1210.4508 (2012).

[31] Marco Battaglieri, talk given at this workshop.

[32] J. W. Price et al. [CLAS Collaboration], Phys. Rev. C 71, 058201 (2005), L. Guo, et al., Phys. Rev. C 76, 025208 (2007). 